\title{
Defect formation in long Josephson junctions
}

\section{Gordeeva, Anna; Pankratov, Andrey}

\section{Published in:}

Physical Review B Condensed Matter

Link to article, DOI:

10.1103/PhysRevB.81.212504

Publication date:

2010

Document Version

Publisher's PDF, also known as Version of record

Link back to DTU Orbit

Citation (APA):

Gordeeva, A., \& Pankratov, A. (2010). Defect formation in long Josephson junctions. Physical Review B Condensed Matter, 81(21), 212504. https://doi.org/10.1103/PhysRevB.81.212504

\section{General rights}

Copyright and moral rights for the publications made accessible in the public portal are retained by the authors and/or other copyright owners and it is a condition of accessing publications that users recognise and abide by the legal requirements associated with these rights.

- Users may download and print one copy of any publication from the public portal for the purpose of private study or research.

- You may not further distribute the material or use it for any profit-making activity or commercial gain

- You may freely distribute the URL identifying the publication in the public portal

If you believe that this document breaches copyright please contact us providing details, and we will remove access to the work immediately and investigate your claim. 


\title{
Defect formation in long Josephson junctions
}

\author{
Anna V. Gordeeva ${ }^{1,2}$ and Andrey L. Pankratov ${ }^{1, *}$ \\ ${ }^{1}$ Institute for Physics of Microstructures, RAS, GSP-105, Nizhny Novgorod 603950, Russia \\ ${ }^{2}$ Department of Physics, B309, Technical University of Denmark, DK-2800 Lyngby, Denmark \\ (Received 14 April 2010; revised manuscript received 31 May 2010; published 22 June 2010)
}

\begin{abstract}
We study numerically a mechanism of vortex formation in a long Josephson junction within the framework of the one-dimensional sine-Gordon model. This mechanism is switched on below the critical temperature. It is shown that the number of fluxons versus velocity of cooling roughly scales according to the power law with the exponent of either 0.25 or 0.5 depending on the temperature variation in the critical current density.
\end{abstract}

DOI: 10.1103/PhysRevB.81.212504

PACS number(s): 03.75.Lm, 05.40.-a, 74.40.-n

There is a well-known experimental fact that annular Josephson junctions (JJs) may trap one or more magnetic flux quanta under cooling below the critical temperature. ${ }^{1,2}$ The nature of this spontaneous trapping is under discussion, particularly when cooling occurs at zero magnetic field. The questions to be answered are how many fluxons and antifluxons are trapped and how their number depends on the cooling rate.

By now a theory explains the spontaneous vortex formation by causality which restricts the possible domain size in the course of the finite in time phase transition. ${ }^{3,4}$ This mechanism is believed to be relevant in various systems: ${ }^{5}$ superfluid transition, ${ }^{6}$ Bose-Einstein condensation, ${ }^{7}$ and cosmic strings. ${ }^{8}$ The theory says that the number of defects at the end of the phase transition scales with the quench time $\tau_{Q}$ (inversely proportional to the cooling rate) according to the power law,

$$
\hat{\xi}=\xi_{0}\left(\frac{\tau_{Q}}{\tau_{0}}\right)^{\sigma}
$$

where $\hat{\xi}$ is the mean distance between defects. An exponent $\sigma$ is of great interest for experimental verification while $\xi_{0}$ and $\tau_{0}$ are related to the specific properties of a system under transition. The scaling, Eq. (1), was shown in the set of experiments, ${ }^{1,2}$ however the value of the exponent was unexpected from the original theory.

Many numerical simulations have been done within the framework of the Ginzburg-Landau model, ${ }^{9-13}$ basically confirming the conclusions of the theory. In Ref. 9, the scaling 0.23-0.33 was obtained for one-dimensional system, twodimensional (2D) systems were considered in Ref. $10(\sigma$ $=0.44-0.79$ ) and Ref. 11 (no scaling was given).

So far the temperature range responsible for fluxon formation is a matter of investigation. ${ }^{12,13}$ Numerical simulations ${ }^{13}$ have been presented to demonstrate that only parameters below $T_{c}$ influence the number of defects. In the Ginzburg-Landau model the phase of the order parameter gets freedom in choosing its value when the module of the order parameter is zero. If the temperature is below $T_{c}$ the kink formation can only happen due to the thermal fluctuations. In the present Brief Report we study a simplified picture in comparison with the Ginzburg-Landau model and use the sine-Gordon model to describe the defect formation. In this model, the dynamics of the module is neglected and the only evolution of the phase is considered, which is the Josephson phase in our case. The freedom for the phase comes in our model from zero value of the critical current at $T$ $=T_{c}$. The sine-Gordon model is quantitatively valid in a narrow temperature range just below the critical temperature $T_{c}$ only ${ }^{14,15}$ that is the region we are interested in. Here one cannot refer to the Kibble-Zurek mechanism anymore, instead the sine-Gordon equation provides an alternative mechanism of defect formation, which we call "the fluctuational mechanism." In the present Brief Report we demonstrate that this simpler model leads to the results that qualitatively agree with the Kibble-Zurek predictions.

The aim of this Brief Report is to investigate whether the fluctuations can lead to a significant fluxon formation with the behavior similar to Kibble-Zurek scenario. The system under study is a long overlap Josephson junction. The annular one-dimensional geometry is also considered as well as the experimental data from Ref. 2 are given for comparison with simulations.

In the experiments ${ }^{2}$ the measured exponent appeared to be twice larger than the initially expected value. ${ }^{1}$ In the original theory such exponent can be obtained for $2 \mathrm{D}$ systems only, ${ }^{10}$ whereas the experimental samples are rather one dimensional (JJs have circumference $500 \mu \mathrm{m}$ and width $4 \mu \mathrm{m}$ ). An explanation was proposed based on the temperature dependence of the critical current of an annular JJ in the vicinity of the critical temperature. It has been shown that the linear dependence leads to $\sigma=0.25,{ }^{1}$ whereas the quadratic law ${ }^{16}$ gives $\sigma=0.5 .^{2}$ From this the conclusion has been drawn that such quadratic dependence is indeed the case of the measured annular JJ. Under this modification the theory fits the Kibble-Zurek picture and is in accordance with the experiment. The resistive sine-Gordon model allows us to implement different expressions for critical current versus temperature and thus to investigate this effect numerically.

One of the disadvantages of the one-dimensional sineGordon model is that in the case of an annular junction the only kink-antikink pairs can be produced whereas in reality one kink is observed undeniably. On the other hand, the use of a linear (free-boundary) one-dimensional sine-Gordon model leads to disappearance of a certain number of kinks at the boundaries. Nevertheless, for junctions with larger lengths, the linear sine-Gordon model allows to clearly distinguish two different temperature dependences of the critical current, which is the most important result of the present Brief Report. 


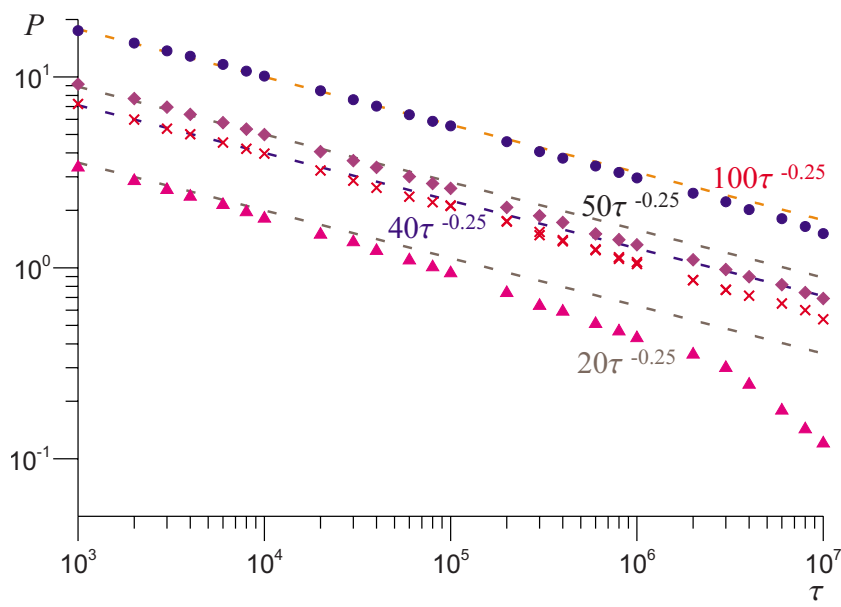

FIG. 1. (Color online) The average number of kinks versus quench time for the linear temperature dependence of the critical current $f(t)=\left[1-T(t) / T_{c}\right]$. The junction lengths are 2000, 1000, $800,400 \lambda_{J}$ (from top to bottom).

The phase evolution, described by the sine-Gordon equation, has a mechanical analog, a string moving in the washboard potential $U(\varphi)=-J_{0} \cos (\varphi)-J \varphi$. In the absence of a bias current $J$ the potential is a simple cosine. To write down the dimensionless sine-Gordon equation let us introduce two characteristic quantities, the Josephson length $\lambda_{J}$ and the plasma frequency $\omega_{p}$, which are time independent,

$$
\lambda_{J}=\sqrt{\frac{\hbar}{2 e L_{0} J_{0}}}, \quad \omega_{p}=\sqrt{\frac{2 e J_{0}}{\hbar C}} .
$$

Here $J_{0}$ is the critical current, $L_{0}$ is the inductance, $C$ is the capacitance, $e$ is the electron charge, and $\hbar$ is the Planck's constant. All values are assumed to be known at the temperature $T_{0}=4.2 \mathrm{~K}$.

After normalization of space to $\lambda_{J}$ and time to $\omega_{p}$ we obtain the following sine-Gordon equation: ${ }^{14,15}$

$$
\varphi_{x x}-\varphi_{t t}-\alpha \varphi_{t}=f(t) \sin (\varphi)+i_{f}(x, t) .
$$

Here $\alpha=\omega_{p} / \omega_{c}$ is the Ohmic loss parameter, $\omega_{c}=2 e J_{0} R / \hbar, R$ is the effective shunt resistance, $f(t)$ is the temperature dependence of the critical current, $\left\langle i_{f}(x, t)\right\rangle=0$, and

$$
\left\langle i_{f}(x, t) i_{f}\left(x+x^{\prime}, t+t^{\prime}\right)\right\rangle=2 \gamma \alpha \delta\left(x^{\prime}\right) \delta\left(t^{\prime}\right),
$$

where $\gamma=2 e k T(t) / \hbar J_{0} \lambda_{J}$ is the dimensionless noise intensity, $T(t)=\left(T_{c}-T_{0}\right) \exp (-t / \tau)+T_{0}$ is the temperature, varying in time, $T_{0}=4.2 \mathrm{~K}$, and $T_{c}$ is the critical temperature of $\mathrm{JJ}$. Boundary conditions for the linear (free-boundary) case are the following:

$$
\varphi_{x}(0, t)=\varphi_{x}(L, t)=0,
$$

where $L$ is the junction length while in the annular case the periodic boundary conditions are used,

$$
\varphi(0, t)=\varphi(L, t), \quad \frac{\partial \varphi(0, t)}{\partial x}=\frac{\partial \varphi(L, t)}{\partial x} .
$$

Thus, we have two time-dependent parameters in the Eq. (3): the critical current and the noise intensity. The resistance

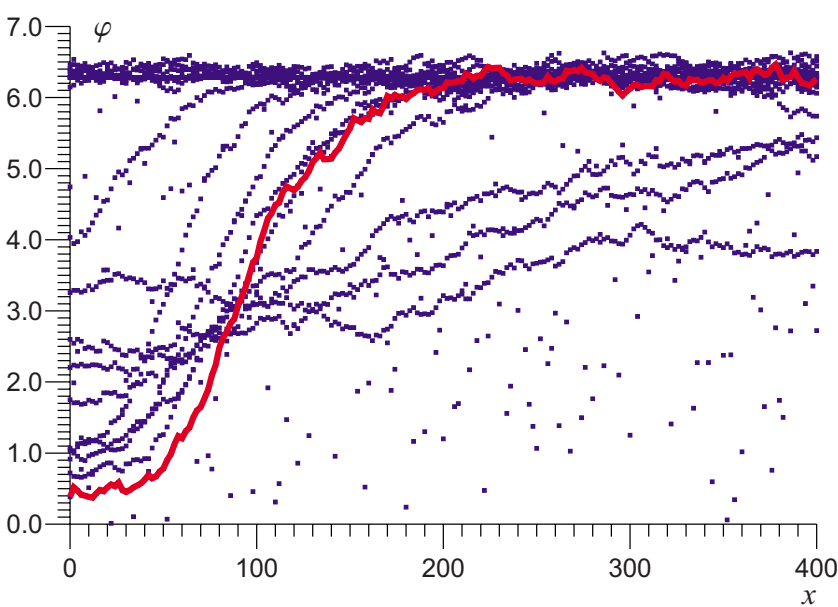

FIG. 2. (Color online) The snapshots of the string evolution during the kink formation, see the explanation in the text.

$R$ is supposed to be equal to the normal-state resistance although this approximation is good below the critical temperature only.

We start the simulations from the temperature $T=T_{c}$, gradually decreasing it: $T(t)=\left(T_{c}-T_{0}\right) \exp (-t / \tau)+T_{0}$. The cooling time $\tau$ is the same as the quenching time. Since at initial moment of time the temperature is equal to the critical temperature, the phase of the order parameter does not exist, which we simulate as an initial random uniform distribution of the phase from 0 to $2 \pi$. In our task the critical current depends on time, so at the initial moment the potential is a flat plane and the potential maxima and minima grow gradually during the evolution. The simulations are performed using the same implicit finite-difference scheme as in Refs. 17-19 with the temporal and spatial steps $\Delta t=\Delta x$ $=0.02-0.2$. The values of parameters in all figures: $\alpha=0.1$, $\gamma=0.00075$ for $T=T_{0}$ (they were calculated for experimental parameters from Ref. 2). The ensemble averaging was performed over 200-1000 realizations.

To describe a transition from the critical temperature to $T_{0}=4.2 \mathrm{~K}$ we take into consideration the temperature dependence of the critical current. Normalized critical current enters the equation as a coefficient $f(t)$ in front of the sine term. Above the critical temperature, the critical current is zero, $f=0$. Below $T_{c}$ the BCS approximation gives linear dependence, Eq. (5), for $f(T)$. But, we also tested a quadratic law, Eq. (6), to see if the substitution of Eq. (5) for Eq. (6) in the Eq. (3) would be enough to change the scaling exponent in two times,

$$
\begin{aligned}
& f(t)=\left[1-T(t) / T_{c}\right], \\
& f(t)=\left[1-T(t) / T_{c}\right]^{2} .
\end{aligned}
$$

Quadratic behavior, Eq. (6), of the critical current below $T_{c}$ was derived from a microscopic model, ${ }^{16}$ in which it was caused by the proximity effect. The results of simulations are presented in Figs. 1 and 3. Here we count all kinks and antikinks whereas in the experiments the only difference between them can be measured. But in the experiments for 


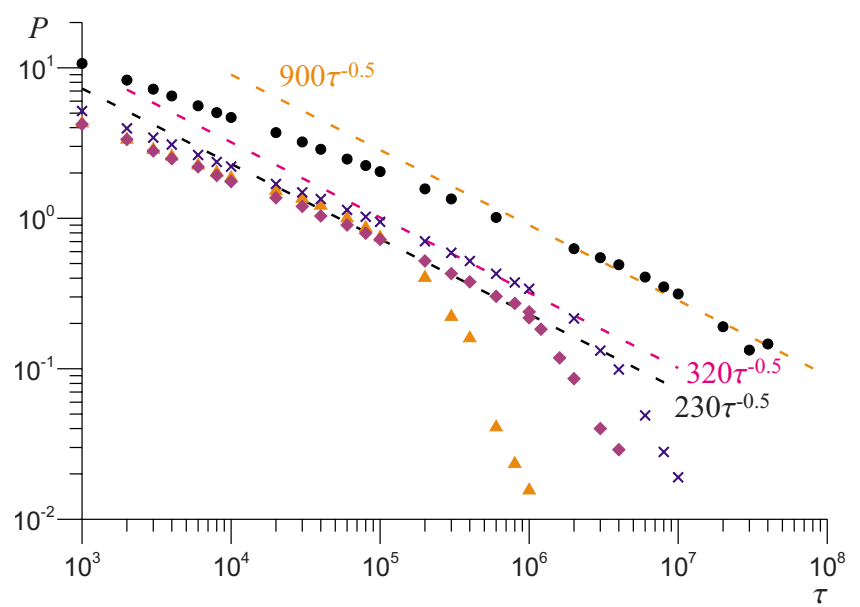

FIG. 3. (Color online) The average number of kinks versus quench time for the quadratic temperature dependence of the critical current $f(t)=\left[1-T(t) / T_{c}\right]^{2}$. The junction lengths are 2000, 1000, $800 \lambda_{J}$ (from top to bottom). By triangles the number of kinks is shown for an annular junction of the length $L=800$.

working range of parameters the appearance of two or more fluxons and antifluxons was unlikely.

In Fig. 1 the typical curves of the kink number versus the quench time are presented for the temperature dependence of the critical current, Eq. (5), and different lengths of the junction. As one can see, at large length $L=2000$, the calculated probability fits well the power-law scale $\tau^{-0.25}$ in almost all range of $\tau$, while certain deviation is observed in the last decade only. With further decrease in the junction length, the deviation becomes more pronounced, however, one may see several steps, which still keep the corresponding $\tau^{-0.25}$ dependence. For $L=1000$ such steps are observed for $\tau$ from $2 \times 10^{4}-1 \times 10^{5}, 2 \times 10^{5}-4 \times 10^{5}, 6 \times 10^{5}-1 \times 10^{7}$. For $L$ $=400$ one can see significant deviation at large $\tau$. This deviation is explained by disappearance of kinks at boundaries of the junction during the kink formation. Such boundary effects are illustrated in Fig. 2. As one can see, from the initial random distribution, the string is formed in the course of time and located around $\varphi=\pi$. After that, the kink is formed around $x=100$ with shorter end tending to $\varphi=0$ and longer end tending to $\varphi=2 \pi$. At a certain moment of time, where the string is shown by red thick solid curve, the kink is actually formed, but it starts to move toward the left boundary $x=0$ and eventually disappears.

In Fig. 3 the typical curves of the kink number versus the quench time are presented for the temperature dependence of the critical current, Eq. (6), and different lengths of the junction. Here three regions are observed. The first one is for the small quench times, where the number of kinks is maximal. Here the number of kinks can be well fitted by $\tau^{-0.4}$, which can easily be distinguished with $\tau^{-0.25}$ of Fig. 1. After this saturation part the most interesting region starts, which can be very well approximated by the linear dependence $\tau^{-0.5}$. In the last region for very large quench times the linear dependence changes its slope roughly to $\tau^{-1.5}$ and we observe a rapid fall of the kink number down to zero. As it has been demonstrated above, the last part is due to disappearance of the kinks at the boundaries. The intermediate region with the

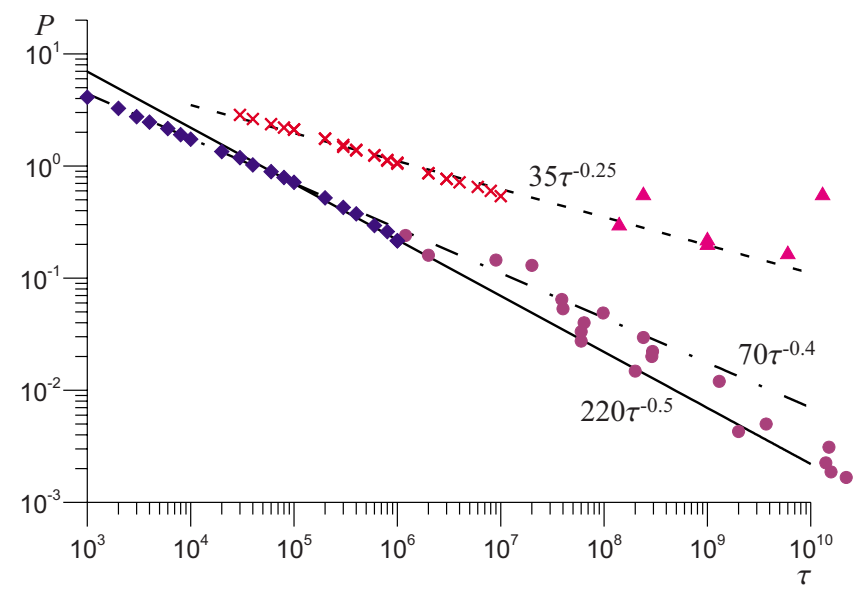

FIG. 4. (Color online) The average number of kinks versus quench time for the linear, Eq. (5), (crosses) and quadratic, Eq. (6), (diamonds) temperature dependences of the critical current. Triangles and circles are experimental data from Refs. 1 and 2.

slope $\tau^{-0.5}$ is rather broad and is on the order of 1.5 decade. Comparing the obtained curves with the number of kinks for an annular junction (which is the same one-dimensional sineGordon model but with periodic boundary conditions), one can see that the fall with $\tau^{-1.5}$ appears earlier, while for small quench times the curves nearly agree, with the annular case giving a bit larger kink number. In the experiments ${ }^{1,2}$ the probability to create single kinks is usually by one to two orders of magnitude larger than the probability to create kink-antikink pairs or double kinks. Therefore, the observed above agreement of the kink number for the linear and the annular cases clearly demonstrates the lack of the considered one-dimensional sine-Gordon model to quantitatively describe the experimental situation. While in the annular model the appearance of single kinks is not allowed due to periodic boundary conditions, the fact of nearly the same number of kinks in the linear case demonstrates very strong trapping of kinks at boundaries.

Nevertheless, it is interesting to plot the kink number for different temperature dependences of the critical current in one figure. This is done in Fig. 4. It is intriguing to see, that the obtained number of kinks by the order of magnitude behaves very similarly to the experimental values (obtained, however, for different junction lengths and quench times), see Fig. 4 of Ref. 2. In spite that in the region of small-tomoderate quench times the kink number is well approximated by the law $\tau^{-0.4}$, while for larger quench times as $\tau^{-0.5}$, the different temperature dependences of the critical current, Eq. (5), (giving $\tau^{-0.25}$ dependence of the kink number) and Eq. (6) can be well distinguished one from another. Continuing further the straight lines, fitting the numerical data, multiplying the time in experiment by $10^{9}$ and placing the experimental data in Fig. 4, one can see that they lie well on the same straight lines. This can be one more justification that different laws of the probability is indeed due to the different temperature dependences of the critical current.

In Refs. 11 and 13 it has been demonstrated that the thin interval of temperatures close to $T_{c}$ is the most crucial for the kink production and the observed number of kinks can sig- 


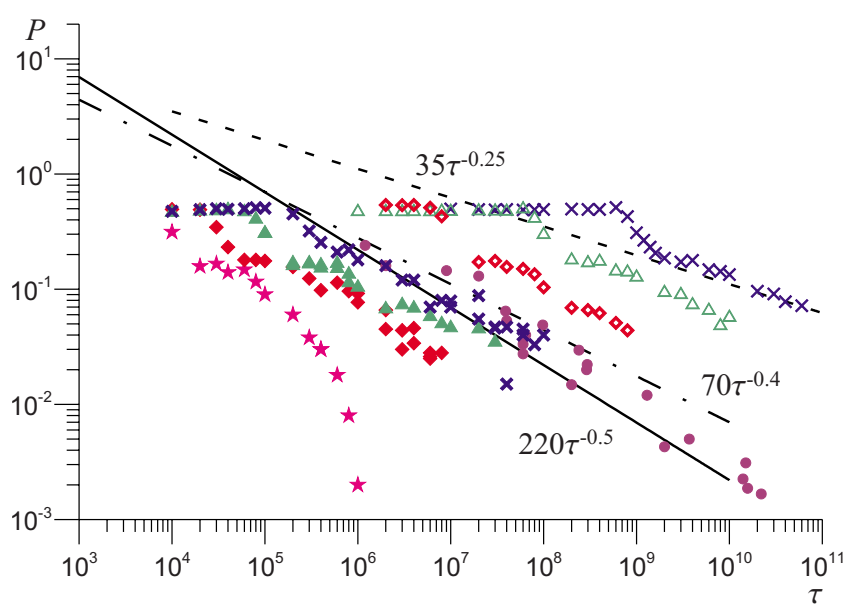

FIG. 5. (Color online) The average number of kinks versus quench time for the linear, Eq. (5), (light symbols) and quadratic, Eq. (6), (dark symbols) temperature dependences of the critical current and different levels of freezing $f(t)=10^{-7}$-crosses, $f(t)=10^{-6}$ — triangles, $f(t)=10^{-5}$ —diamonds, and $f(t)=10^{-4}$ — stars. Circles- the experimental data from Ref. 2.

nificantly decrease with time due to annihilation between kinks and antikinks, and also due to escape of unbounded vortices out of the sample. This decrease in the kink number can be easily checked by choosing of different levels of the smooth (exponential) cooling $f(t)=b_{0}$, so after this boundary is reached, the abrupt freezing $T(t)=0, f(t)=1$ occurs. The number of single kinks (the number of kink-antikink pairs is not taken into account), counted after such procedure, is pre- sented in Fig. 5. One can see that unlike Fig. 4, here nearly the same kink number is reached for the junction length $L$ $=80$ and $f(t)=10^{-7}$ (crosses). Further increase in the boundary of the smooth cooling leads to decrease in the kink number, nevertheless, for the case, Eq. (6), the kink number follows the dependence $\tau^{-0.5}$ in two to three orders of magnitude until $f(t)=10^{-5}$ (diamonds). Fitting by the case $\tau^{-0.25}$ for Eq. (5) is worse but improves with the decrease in calculation steps $\Delta t$ and $\Delta x$. Therefore, this result allows us to hope that the use of more advanced models (either sineGordon or Ginzburg-Landau two-dimensional annular systems, allowing both flux conservation and appearance of single vortices during the quench process) will finally lead to quantitative agreement between the experimental and numerical results.

In the present Brief Report the mechanism of vortex formation in a long Josephson junction has been studied numerically within the framework of the one-dimensional sineGordon model with white noise. It has been demonstrated that the number of fluxons versus velocity of cooling roughly scales according to the power law with the exponent of either 0.25 or 0.5 depending on the temperature variation in the critical current. The results of simulations qualitatively agree both with the experimental results of Ref. 2 and with the theory.

The authors wish to thank J. Mygind, R. Monaco, M. P. Soerensen and M. R. Samuelsen for discussions and useful comments. The work has been supported by RFBR (Projects No. 09-02-00491 and No. 08-02-97033).

\footnotetext{
*alp@ipm.sci-nnov.ru

${ }^{1}$ R. Monaco, J. Mygind, and R. J. Rivers, Phys. Rev. B 67, 104506 (2003).

${ }^{2}$ R. Monaco, M. Aaroe, J. Mygind, R. J. Rivers, and V. P. Koshelets, Phys. Rev. B 74, 144513 (2006).

${ }^{3}$ T. W. B. Kibble, Phys. Rep. 67, 183 (1980).

${ }^{4}$ W. H. Zurek, Phys. Rep. 276, 177 (1996).

${ }^{5}$ T. Kibble, Phys. Today 60(9), 47 (2007).

${ }^{6}$ V. B. Efimov, O. J. Griffiths, P. C. Hendry, G. V. Kolmakov, P. V. E. McClintock, and L. Skrbek, Phys. Rev. E 74, 056305 (2006); L. Mathey and A. Polkovnikov, Phys. Rev. A 81, 033605 (2010).

${ }^{7}$ C. N. Weiler, T. W. Neely, D. R. Scherer, A. S. Bradley, M. J. Davis, and B. P. Anderson, Nature (London) 455, 948 (2008); C. Lee, Phys. Rev. Lett. 102, 070401 (2009).

${ }^{8}$ J. J. Blanco-Pillado, K. D. Olum, and A. Vilenkin, Phys. Rev. D 76, 103520 (2007); A. Rajantie, ibid. 79, 043515 (2009).

${ }^{9}$ P. Laguna and W. H. Zurek, Phys. Rev. D 58, 085021 (1998).

${ }^{10}$ A. Yates and W. H. Zurek, Phys. Rev. Lett. 80, 5477 (1998).
}

${ }^{11}$ M. Ghinovker, B. Ya. Shapiro, and I. Shapiro, Europhys. Lett. 53, 240 (2001).

${ }^{12}$ L. M. A. Bettencourt, N. D. Antunes, and W. H. Zurek, Phys. Rev. D 62, 065005 (2000).

${ }^{13}$ N. D. Antunes, P. Gandra, and R. J. Rivers, Phys. Rev. D 73, 125003 (2006).

${ }^{14}$ K. K. Likharev, Dynamics of Josephson Junctions and Circuits (Gordon and Breach, New York, 1986).

${ }^{15}$ A. Barone and G. Paterno, Physics and Applications of the Josephson Effect (Wiley, New York, 1982).

${ }^{16}$ A. A. Golubov, E. P. Houwman, J. G. Gijsbertsen, V. M. Krasnov, J. Flokstra, H. Rogalla, and M. Yu. Kupriyanov, Phys. Rev. B 51, 1073 (1995).

${ }^{17}$ K. G. Fedorov and A. L. Pankratov, Phys. Rev. B 76, 024504 (2007).

${ }^{18}$ A. L. Pankratov, Appl. Phys. Lett. 92, 082504 (2008).

${ }^{19}$ K. G. Fedorov and A. L. Pankratov, Phys. Rev. Lett. 103, 260601 (2009). 